\title{
Drug Reaction with Eosinophilia and Systemic Symptoms Associated with a Vancomycin- Impregnated Spacer
}

\author{
Blake Michael Bodendorfer, BA ${ }^{1}$ Emily Fahrig Cole, $\mathrm{MPH}^{2}$ Stephanie J. Carstens, $\mathrm{MD}^{3}$ \\ Seth L. Sherman, MD ${ }^{1}$ Natraj Katta, MD ${ }^{2}$
}

${ }^{1}$ Department of Orthopaedic Surgery, University of Missouri, Columbia, Missouri

2 Department of Medicine, University of Missouri, Columbia, Missouri

${ }^{3}$ Department of Dermatology, University of Missouri,

Columbia, Missouri

Address for correspondence Blake Michael Bodendorfer, BA, University of Missouri School of Medicine, MA215 Medical Sciences Building, Columbia, MO 65212

J Knee Surg Rep 2015;1:31-34.

\begin{abstract}
Drug reaction with eosinophilia and systemic symptoms (DRESS) is a severe adverse drug reaction with a mortality rate near $10 \%$ characterized by erythematous cutaneous eruption, multiorgan involvement, and hypereosinophilia. This case report highlights a patient who developed DRESS in the setting of intravenous vancomycin and a vancomycin and tobramycin-impregnated (PROSTALAC, DePuy Synthes, Warsaw, IN) functional spacer. The spacer was placed during the revision of a left total knee replacement complicated by a septic joint. To our knowledge, this is the third reported case of DRESS associated with vancomycin-impregnated spacers. As DRESS is a potentially life-threatening reaction and the principal treatment is withdrawal of the precipitating drug, it is important to consider this clinical entity and discontinue all forms of the causative agent. Patients with DRESS may develop a diffuse cutaneous eruption, respiratory failure, acute kidney injury, elevated liver enzymes, lymphadenop-

\section{Keywords}

- vancomycin

- antibiotic spacer

- rash

- DRESS syndrome athy, and/or hemodynamic instability following intravenous infusion of antibiotics or placement of antibiotic-impregnated spacers, beads, or bone cement. Interestingly, this patient had a prominent and progressive erythematous eruption surrounding the left knee that improved after operative removal of the spacer. At 6 week hospital follow-up, the patient's systemic signs and symptoms had essentially resolved.
\end{abstract}

Drug reaction with eosinophilia and systemic symptoms (DRESS) is a severe drug reaction characterized by cutaneous eruption, hypereosinophilia, and multiorgan involvement. ${ }^{1}$ The term was first used by Bocquet et al in 1996 to classify various cutaneous drug reactions with similar pathophysiological mechanisms that mimic malignant lymphoma. ${ }^{2}$ Visceral manifestations may include hepatic, renal, and pulmonary sequelae, ${ }^{3}$ and mortality rates as high as $10 \%$ have been reported. ${ }^{4,5}$ Common precipitants include anticonvulsants (phenytoin, phenobarbital, carbamazepine, lamotrigine), allopurinol, nitrofurantoin, and leflunomide. Less frequently, antibiotics have been associated with the development of DRESS. In addition, there is an association between reactivation of human herpesvirus- 6 and increased severity of organ involvement in DRESS. ${ }^{6-8}$ Here, we report a case of DRESS with associated acute kidney injury, elevated liver enzymes, facial swelling, and erythematous cutaneous eruption affecting $90 \%$ of body surface area that was precipitated by intravenous vancomycin and a received

June 17, 2014

accepted after revision

November 6, 2014

published online

February 11, 2015
DOI http://dx.doi.org/

10.1055/s-0034-1399761. ISSN 2326-2729.
Copyright $\odot 2015$ by Thieme Medical Publishers, Inc., 333 Seventh Avenue, New York, NY 10001, USA. Tel: +1(212) 584-4662.
License terms

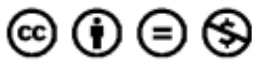


vancomycin and tobramycin-impregnated (PROSTALAC) functional knee spacer used for revision of a total knee replacement complicated by prosthetic knee infection.

\section{Case}

A 65-year-old Caucasian male patient with a medical history of chronic kidney disease and osteoarthritis, but no prior allergic reactions, underwent left total knee replacement complicated by prosthetic knee infection approximately 6 years after original placement. The cause of the prosthetic joint infection was not identified. During the patient's clinical course, the knee was aspirated, and no growth was ever observed via culture. Additionally, blood cultures were also negative when the clinical symptomatology of DRESS syndrome appeared. However, his synovial WBC count was 37,500 with $83 \%$ neutrophils, which was very concerning for infection based on accepted criteria. ${ }^{9}$ Following irrigation, debridement, and removal of the infected device, a PROSTALAC functional spacer (impregnated with vancomycin and tobramycin) was surgically placed. The patient tolerated the procedure well and was concurrently started on a 6-week course of outpatient intravenous vancomycin.

One day after completing the 6-week course of intravenous vancomycin, the patient developed chills, a fever (maximum $38.7^{\circ} \mathrm{C}$ ), and an erythematous, extremely pruritic eruption on the posterior neck which progressed within 5 days to involve his back, arms, and legs. Despite diphenhydramine and prednisone prescribed after emergency room evaluation for presumed allergic reaction of uncertain etiology, the rash progressed to involve his face and approximately $90 \%$ of his body surface area. In addition, he developed swelling of his periorbital region and lips, painful tingling in areas with rash, myalgias, and fatigue that prompted hospital admission 5 days after the initial onset of skin symptoms.

On admission, vital signs included a temperature of $39.8^{\circ} \mathrm{C}$, pulse of 128 beats per minute, respiratory rate of 25 breaths per minute, and blood pressure of $159 / 80 \mathrm{~mm} \mathrm{Hg}$. Physical examination revealed prominent erythematous perifollicular macules and papules coalescing into patches and plaques on bilateral thighs and lower legs, as well as confluent and diffuse facial and truncal erythema that was warm to touch. The facial edema was most prominent in the periorbital region and his lips. There was no palpable cervical or axillary lymphadenopathy and no evidence by examination for cardiac inflammation, hepatosplenomegaly, or other edema. The left lower extremity examination revealed a well-healed midline scar over the knee and reduced range of motion for both flexion and extension, but strength, sensation, and peripheral pulses were all normal. $\mathrm{X}$-rays of the left knee showed the total knee arthroplasty in good position with the spacer present.

Laboratory evaluation revealed leukocytosis (19,300/L) with $10 \%$ eosinophilia $(1,930 / \mathrm{L})$, creatinine of $1.47 \mathrm{mg} / \mathrm{dL}$ (baseline $1.21 \mathrm{mg} / \mathrm{dL}$ ), elevated alanine aminotransferase of $57 \mathrm{U} / \mathrm{L}$, and lactic acid of $3 \mathrm{mmol} / \mathrm{L}$. The urinalysis was benign. Inpatient workup for presumed adverse drug reaction included antistreptolysin titers, rubeola IgG, herpes simplex virus 1 and $2 \operatorname{IgG}$ and $\operatorname{IgM}$, coxsackievirus polymerase chain reaction
(PCR), cytomegalovirus PCR, human herpesvirus 6, hepatitis $\mathrm{A}, \mathrm{B}$, and $\mathrm{C}$ panel, rapid plasma reagin, Borrelia burgdorferi antibodies, and cultures of blood and urine which were all nondiagnostic. Vancomycin level was less than $1.7 \mu \mathrm{g} / \mathrm{mL}$. The patient's skin eruption continued to progress and became more violaceous during the course of the hospitalization, particularly in the distal extremities.

Inpatient dermatology consultation noted a diffuse, perifollicular erythematous eruption-most prominently of bilateral thighs-confluent and diffuse truncal erythema, absolute eosinophilia, acute kidney injury, elevated liver enzymes, and facial edema consistent with the diagnosis of DRESS. The cutaneous eruption was prominent surrounding his left knee (-Fig. 1). Because his clinical picture was enough for diagnosis, biopsy was not completed. It was recommended that the vancomycinimpregnated antibiotic spacer be removed, as it was thought to be contributing to the patient's current clinical state.

After 16 days of appearance of symptoms ( 8 weeks and 3 days following PROSTALAC implantation), the patient underwent surgical removal of the vancomycin-impregnated spacer and revision total left knee arthroplasty. The medical team opted to wait 16 days to optimize kidney function before surgery. He tolerated the procedure well. On postoperative day 2 , the skin symptoms were nearly resolved with the exception of a mild macular erythema of his lower extremities, most prominent near the left knee. In addition, he had mild superficial desquamation of his face, back, and left ankle. He was discharged on postoperative day 3 with topical triamcinolone cream.

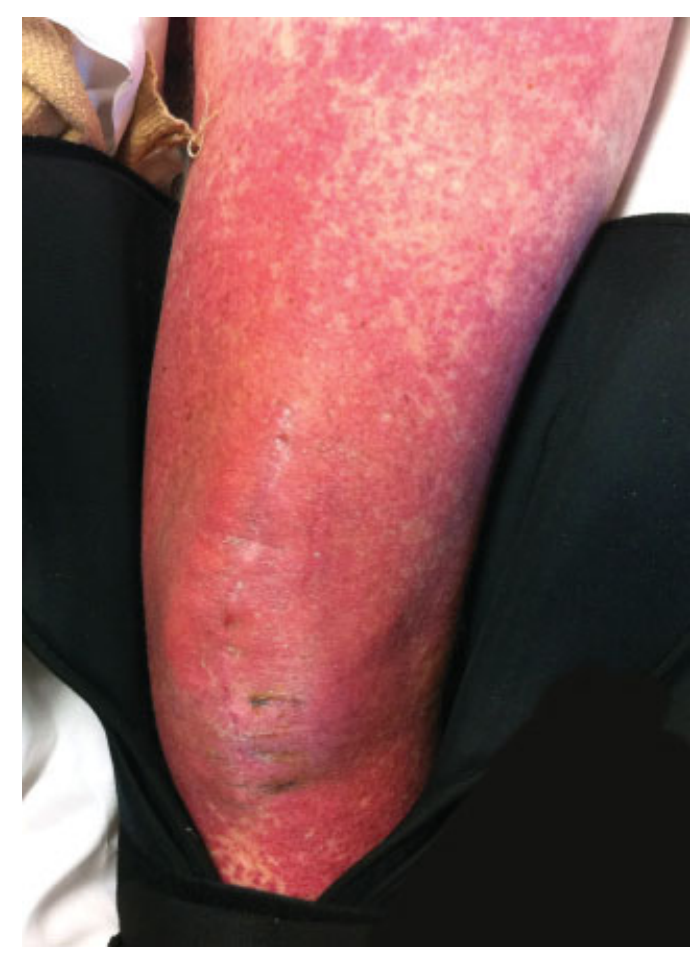

Fig. 1 Cutaneous eruption of left knee with implanted vancomycin and tobramycin-impregnated (PROSTALAC) functional knee spacer. The eruption can be described as erythematous and perifollicular macules and papules that coalesce into confluent patches and plaques. 
At 2 weeks outpatient dermatology follow-up, his leukocyte count, renal function, and liver enzymes had all returned to baseline, and his skin continued to exfoliate at the distal extremities, including palms and soles, as expected for resolution of the rash. At 6 weeks and 6 months outpatient orthopedic surgery follow-ups, the patient's systemic signs and symptoms had essentially resolved. The patient was satisfied with his final outcome. His 6 month knee injury and osteoarthritis outcome score (KOOS) activities of daily living (ADL) score was 86.76, KOOS pain 69.44, KOOS quality of life (QOL) 43.75, KOOS sports and recreational activities (SRA) 80.00 , and KOOS symptoms 67.86. All KOOS scores are out of a maximum of 100 . The mean KOOS scores for an age and gender-matched cohort from the general population are as follows: ADL 86.3, pain 87.7, QOL 78.9, SRA 72.6, and symptoms $88.4 .^{10}$ He will be followed clinically in 2-month intervals, with judicious clinical and laboratory workup if concerning symptoms of prosthetic joint infection arise.

\section{Discussion}

We report a case of DRESS precipitated by a vancomycin and tobramycin-impregnated (PROSTALAC) functional knee spacer. The association between intravenous vancomycin and DRESS has previously been described. ${ }^{11-20}$ The most common antecedent infections include endocarditis ${ }^{13,19}$ and osteomyelitis. ${ }^{14,16,17,21}$ DRESS is an adverse drug reaction with a long latency period (1-8 weeks) with the majority presenting between 4 and 6 weeks following initiation of antibiotic therapy. In most cases, withdrawal of the offending agent and administration of corticosteroids were sufficient treatment, although cyclosporine has also been used with good results. ${ }^{12}$ Living-donor liver transplantation was required in one case. ${ }^{21}$

To our knowledge, this is the third reported case of DRESS resulting from vancomycin-impregnated orthopedic spacers and intravenous vancomycin. In both cases previously reported, ${ }^{20,22}$ appearance of a diffuse erythematous rash, respiratory failure, hemodynamic instability, and acute kidney injury which required dialysis occurred 2 weeks following placement of the antibiotic spacer. Vesicular eruptions were also described in one case. ${ }^{20}$ Both cases responded well to high-dose intravenous steroids ( $1 \mathrm{~g} / \mathrm{d}$ vs. $1 \mathrm{~g} / 12$ hours) with complete resolution of systemic and dermatologic symptoms after surgical removal of the antibiotic spacer. ${ }^{18,22}$

In contrast to previously reported cases, our patient had a less severe hospital course, demonstrated by the lack of respiratory failure and hemodynamic instability. Some literature suggests a correlation between human herpesvirus- 6 reactivation and severe organ involvement in DRESS. ${ }^{6-8,15}$ We did not observe reactivation of human herpesvirus- 6 in our patient, and this may have been beneficial for this patient's recovery. However, neither of the other two reported cases $^{20,22}$ commented on human herpesvirus- 6 reactivation.

Although a microbial etiology of the patient's presentation was certainly considered, we believe the patient's presentation was due to vancomycin for multiple reasons. First, a 6-week course of vancomycin was given, and the patient presented with his symptoms after the antibiotics were discontinued; reasonably, we conclude that systemic infection would be unlikely in this context. Next, multiple blood cultures were negative, which rule down systemic inflammatory response syndrome or sepsis as an etiology. Finally, the patient's dramatic response to the antibiotic spacer removal served as confirmation of our hypothesis. While other etiologies are possible, we believe DRESS is the most likely cause.

Systemic corticosteroids have anecdotally been shown to benefit patients with severe renal involvement (demonstrated by creatinine $>150 \%$ of baseline, proteinuria, or hematuria) or pulmonary involvement (demonstrated by hypoxemia, dyspnea, or abnormal chest radiograph), although no randomized trials have been conducted. While prompt administration of oral steroids before this patient's hospital admission may have contributed to a less severe hospital course, the benefit of systemic corticosteroids in patients without severe organ involvement $\mathrm{t}^{23,24}$ and with severe liver involvement ${ }^{25}$ has not been proven. Most patients with DRESS are arbitrarily treated with systemic corticosteroids, and while it may not be harmful to patient outcome, this may be unnecessary. 3,24,26 Additionally, systemic steroid administration is an established risk factor for prosthetic joint infection, and may worsen outcome in patients with active infection. ${ }^{27}$

In all three cases, placement of the vancomycin-impregnated spacer was followed by long-term administration of intravenous vancomycin. It is possible that the intravenous vancomycin rather than the spacer was the precipitant for the development of DRESS. However, our patient's exanthem was brightly erythematous and coalescent in the area surrounding the left knee containing the spacer. This was also an area of skin that was the slowest to resolve. The dermatologic and systemic manifestations resolved completely in all three cases with steroids, discontinuation of intravenous vancomycin, and surgical removal of the vancomycin-impregnated spacer.

Our case highlights the importance of possessing a high index of clinical suspicion for drug-related hypersensitivity reactions following placement of antibiotic-impregnated spacers, beads, and bone cement. In the case of this patient, a drug reaction related to vancomycin was strongly suspected and intensity of the rash over the left knee containing the spacer suggested that both intra-articular and intravenous vancomycin were causative. Although intravenous administration generates higher serum levels than antibiotic-loaded prostheses, Masri et al have demonstrated that the majority of patients with PROSTALAC placement will have measurable intra-articular levels of the drug at a mean of 118 days. ${ }^{28}$ This patient became symptomatic 44 days after the spacer was implanted. Other antibiotic-impregnated spacers, beads, and bone cement have been associated with postoperative complications, including isolated cutaneous eruption, ${ }^{29}$ hypersensitivity reactions, ${ }^{21}$ drug fever, ${ }^{30}$ and acute kidney injury. ${ }^{31,32}$

DRESS is extremely uncommon, with an estimated incidence of 1 out of every 10,000 individuals exposed to an implicated medication. ${ }^{5}$ In addition, due to the idiosyncratic nature of the reaction it is nearly impossible to predict which patients will develop DRESS nor the severity of disease. 
Because DRESS and its sequelae are potentially fatal, rapid identification and removal of all forms of the causative drug are necessary to reduce associated morbidity and mortality. DRESS should be suspected in any patient who develops cutaneous eruption, eosinophilia, respiratory symptoms, renal failure, elevated liver enzymes, or lymphadenopathy following surgical placement of a vancomycin-impregnated orthopedic spacer, beads, or bone cement.

\section{References}

1 Walsh SA, Creamer D. Drug reaction with eosinophilia and systemic symptoms (DRESS): a clinical update and review of current thinking. Clin Exp Dermatol 2011;36(1):6-11

2 Bocquet H, Bagot M, Roujeau JC. Drug-induced pseudolymphoma and drug hypersensitivity syndrome (Drug Rash with Eosinophilia and Systemic Symptoms: DRESS). Semin Cutan Med Surg 1996; 15(4):250-257

3 Chen YC, Chiu HC, Chu CY. Drug reaction with eosinophilia and systemic symptoms: a retrospective study of 60 cases. Arch Dermatol 2010;146(12):1373-1379

4 Ghislain PD, Roujeau JC. Treatment of severe drug reactions: Stevens-Johnson syndrome, toxic epidermal necrolysis and hypersensitivity syndrome. Dermatol Online J 2002;8(1):5

5 Tas S, Simonart T. Management of drug rash with eosinophilia and systemic symptoms (DRESS syndrome): an update. Dermatology 2003;206(4):353-356

6 Shiohara T, Inaoka M, Kano Y. Drug-induced hypersensitivity syndrome (DIHS): a reaction induced by a complex interplay among herpesviruses and antiviral and antidrug immune responses. Allergol Int 2006;55(1):1-8

7 Gentile I, Talamo M, Borgia G. Is the drug-induced hypersensitivity syndrome (DIHS) due to human herpesvirus 6 infection or to allergy-mediated viral reactivation? Report of a case and literature review. BMC Infect Dis 2010;10:49

8 Neuman MG, McKinney KK, Nanau RM, et al. Drug-induced severe adverse reaction enhanced by human herpes virus- 6 reactivation Transl Res 2013;161(5):430-440

9 Ghanem E, Parvizi J, Burnett RS, et al. Cell count and differential of aspirated fluid in the diagnosis of infection at the site of total knee arthroplasty. J Bone Joint Surg Am 2008;90(8):1637-1643

10 Paradowski PT, Bergman S, Sundén-Lundius A, Lohmander LS, Roos EM. Knee complaints vary with age and gender in the adult population. Population-based reference data for the Knee injury and Osteoarthritis Outcome Score (KOOS). BMC Musculoskelet Disord 2006; 7:38

11 Díaz-Mancebo R, Costero-Fernández O, Vega-Cabrera C, et al. Dress syndrome and acute tubulointerstitial nephritis after treatment with vancomycin and beta-lactams. Case report and literature review. Nefrologia 2012;32(5):685-687

12 Zuliani E, Zwahlen H, Gilliet F, Marone C. Vancomycin-induced hypersensitivity reaction with acute renal failure: resolution following cyclosporine treatment. Clin Nephrol 2005;64(2): 155-158

13 Yazganoglu KD, Ozkaya E, Ergin-Ozcan P, Cakar N. Vancomycininduced drug hypersensitivity syndrome. J Eur Acad Dermatol Venereol 2005;19(5):648-650
14 Kwon HS, Chang YS, Jeong YY, et al. A case of hypersensitivity syndrome to both vancomycin and teicoplanin. J Korean Med Sci 2006;21(6):1108-1110

15 Tamagawa-Mineoka R, Katoh N, Nara T, Nishimura Y, Yamamoto S, Kishimoto S. DRESS syndrome caused by teicoplanin and vancomycin, associated with reactivation of human herpesvirus-6. Int J Dermatol 2007;46(6):654-655

16 Vauthey L, Uçkay I, Abrassart S, et al. Vancomycin-induced DRESS syndrome in a female patient. Pharmacology 2008;82(2):138-141

17 O'Meara P, Borici-Mazi R, Morton AR, Ellis AK. DRESS with delayed onset acute interstitial nephritis and profound refractory eosinophilia secondary to Vancomycin. Allergy Asthma Clin Immunol 2011;7:16

18 Schnetzke U, Bossert T, Scholl S, Freesmeyer M, Hochhaus A, La Rosée P. Drug-induced lymphadenopathy with eosinophilia and renal failure mimicking lymphoma disease: dramatic onset of DRESS syndrome associated with antibiotic treatment. Ann Hematol 2011;90(11):1353-1355

19 Cariou ME, Kaabar M, Cailliez P, et al. Vancomycin-induced DRESS syndrome: a case report [in French]. Ann Biol Clin (Paris) 2013; 71(2):190-195

20 Azubike E, Akhil V, Margaret W, et al. Vancomycin induced DRESS syndrome: An unusual cause of life threatening stridor and bronchoconstriction. Am Thor Soc 2010:A3081

21 Song EK, Seon JK, Jeong MS. Delayed-type hypersensitivity reaction to piperacillin/tazobactam in a patient with an infected total knee replacement. J Bone Joint Surg Br 2010;92(11):1596-1599

22 Bajaj P, Prematta MJ, Ghaffari G. A sixty-five-year-old man with rash, fever, and generalized weakness. Allergy Asthma Proc 2011; 32(1):e1-e3

23 Uhara H, Saiki M, Kawachi S, Ashida A, Oguchi S, Okuyama R. Clinical course of drug-induced hypersensitivity syndrome treated without systemic corticosteroids. J Eur Acad Dermatol Venereol 2013;27(6):722-726

24 Um SJ, Lee SK, Kim YH, et al. Clinical features of drug-induced hypersensitivity syndrome in 38 patients. J Investig Allergol Clin Immunol 2010;20(7):556-562

25 Davern TJ. Drug-induced liver disease. Clin Liver Dis 2012;16(2): 231-245

26 Cacoub P, Musette P, Descamps V, et al. The DRESS syndrome: a literature review. Am J Med 2011;124(7):588-597

27 Berbari EF, Hanssen AD, Duffy MC, et al. Risk factors for prosthetic joint infection: case-control study. Clin Infect Dis 1998;27(5): 1247-1254

28 Masri BA, Duncan CP, Beauchamp CP. Long-term elution of antibiotics from bone-cement: an in vivo study using the prosthesis of antibiotic-loaded acrylic cement (PROSTALAC) system. J Arthroplasty 1998;13(3):331-338

29 Williams B, Hanson A, Sha B. Diffuse Desquamating Rash Following Exposure to Vancomycin-Impregnated Bone Cement. Ann Pharmacother 2014;48(8):1061-1065

30 Park HB, Choi JS, Park SH, Kee WJ, Koh YI. Drug fever due to piperacillin/tazobactam loaded into bone cement. J Korean Med Sci 2011;26(2):301-303

31 Patrick BN, Rivey MP, Allington DR. Acute renal failure associated with vancomycin- and tobramycin-laden cement in total hip arthroplasty. Ann Pharmacother 2006;40(11):2037-2042

32 McGlothan KR, Gosmanova EO. A case report of acute interstitial nephritis associated with antibiotic-impregnated orthopedic bone-cement spacer. Tenn Med 2012;105(9):37-40, 42 